\title{
Adaptive gamification in education: A literature review of current trends and developments
}

\author{
Stuart Hallifax ${ }^{10000-0002-2743-9884]}$, Audrey Serna ${ }^{20000-0003-1468-9761]}$, \\ Jean-Charles Marty ${ }^{30000-0003-1823-8415]}$, and Elise \\ Lavoué ${ }^{1[0000-0002-2659-6231]}$ \\ 1 University of Lyon, University Jean Moulin Lyon 3, iaelyon school of Management, \\ CNRS, LIRIS UMR5205 F-69621 \\ 2 INSA de Lyon - CNRS, LIRIS UMR5205 F-69621 \\ 3 Université de Savoie Mont Blanc - CNRS, LIRIS UMR5205 F-69621 \\ name.surname@liris.cnrs.fr
}

\begin{abstract}
Gamification, the use of game elements in non-game settings, is more and more used in education to increase learner motivation, engagement, and performance. Recent research in the gamification field suggests that to be effective, the game elements should be tailored to learners. In this paper, we perform an in-depth literature review on adaptive gamification in education in order to provide a synthesis of current trends and developments in this field. Our literature review addresses 3 research questions: (1) What are the current kinds of contributions to the field? (2) What do the current contributions base their adaptation on, and what is the effect of this adaptation on the gamified system? (3) What is the impact of the adaptive gamification, and how is this impact measured? We also provide future research guidelines in the form of three needs that should be fulfilled for exploring this field.
\end{abstract}

Keywords: Gamification · Education · Adaptation

\section{Introduction}

Gamification, defined as the use of game elements in non-game contexts [12], has been used for close to ten years in educational settings to increase learner performance, motivation, or engagement $[1,24,27]$. Recent studies conducted in other contexts such as health [33] and sport [26] on the effects of gamification show that to be effective, gamification should be tailored to users. In education, research on adaptation has mainly concerned educational content and its adaptation to learners and context. It is a well explored research topic [8] that has been shown to be effective. Adaptive gamification in education attempts to leverage both of these concepts in order to provide a better learner experience. It is therefore important to take a step back and analyse how game elements can be adapted to learners in educational contexts. In this paper, we review the research on adaptive gamification in education and present the results of our analysis. In order to examine the current state of research in this field, and to 
understand how adaptive gamification is applied in education, we performed a literature review based on twenty papers. Through our review, we highlight the advances in the field and limitations that need to be addressed. Our review aims to answer the following questions:

- What are the current kinds of contributions to the field of adaptive gamification in education? We distinguish three kinds of contributions: 1) preliminary research on recommendations for game elements adapted to learner profiles, 2 ) technical contributions on architectures that have not been tested yet and 3) studies that look at the impact of adaptive gamification that make use of such architectures, and that provide valuable results into this research approach. The analysis of these three contribution types show the maturity of this field.

- What do the current contributions base their adaptation on, and what is the effect of this adaptation on the gamified system? We clearly distinguish static (i.e. initial) adaptation and dynamic adaptation, that rely on different kinds of information, such as player types or interaction traces.

- What is the impact of the adaptive gamification and how is this impact measured? We identify studies conducted on short and on long terms, as results obtained may depend on the duration. We also distinguish studies according to the adaptation mechanism used (static or dynamic).

In this paper we first present our literature review process in section 2 . Then, in section 3 we present three parts, each part being dedicated to a research question. We finally provide future directions for research in this field in section 4, by pointing out three needs that future research in this field should aim to resolve: the need for richer learner models, the need to explore different adaptation methods, and the need for more structured studies.

\section{Literature review process}

Our structured literature review process was based on the guidelines and processes described in $[38,39]$. First, we defined our review scope, by specifying our research questions and therefore explicating our search query (we explain this more in detail in section 2.1). Then, we ran our search query and filtered the papers that did not fit our review scope (see section 2.2).

\subsection{Defining our review scope}

We are interested in the current state of adaptive gamification in education research. We studied what exactly is adapted, and what characteristics or variables are used to tailor learner experience. This lead us to define our three research

questions. We then clearly defined our search terms, as to cover the topic of adaptive gamification in education. More specifically we used the search query:

(gamif*) AND (learning OR education OR teaching) AND (adapt* OR tailor* OR personali*) 
The first part of our query (i.e. gamif*) was used to capture all terms that start with "gamif" (i.e. gamification, gamified etc.). Note that the queries "gamif*" and "gamif" were used depending on the capabilities of the search engines used as some allowed for wildcard characters and others not. After testing different permutations of "teaching words" we settled on "Learning" "Education" and "Teaching" (when we added alternatives such as "learn" or "learner" the result count did not change, so we stuck with a more focused approach). Finally for the adaptive part, we had a similar reasoning as with "gamif". The three base words ("adapt", "tailor" and "personali") allowed us to capture the different keywords used to describe these works (and also allow for regional variants such as the British "personalised" versus the American "personalized").

\subsection{Paper search \& filter}

We ran our search query on the major scientific digital libraries (ACM, IEEE, Science Direct, Springer) and Google Scholar. Due to the fairly large nature of our search query, we received a large number of initial hits (370 papers, see table 1 ), which lead to a rigorous filtration process in order to remove false hits.

Table 1. Number of papers before and after content filtering. The number of papers excluded is given for each filtration step.

\begin{tabular}{|c|c|c|c|c|c|}
\hline \multirow{2}{*}{$\begin{array}{l}\text { Filtration step } \\
\text { Keyword query }\end{array}$} & \multicolumn{5}{|c|}{$\begin{array}{c}\text { Source } \\
\text { ACM IEEE Science Direct Springer Google Scholar }\end{array}$} \\
\hline & 64 & 94 & 17 & 35 & 160 \\
\hline Removed - format & 18 & 8 & 1 & 2 & 49 \\
\hline Removed - scope & 41 & 79 & 13 & 26 & 74 \\
\hline Removed - duplicate & 2 & 1 & 1 & 1 & 34 \\
\hline Final count & 3 & 6 & 2 & 6 & 3 \\
\hline
\end{tabular}

Papers were first reviewed by scanning the keywords and title, then the abstract, and finally the full text if the paper was not excluded from the previous two steps. Papers were then excluded for the following reasons:

- Format: Results that were either abstracts, preview content, posters or workshop papers were removed. We made this decision so that we only studied mature works. Finally, we also removed papers that were not in English (many of the results from Google Scholar had English abstracts or titles, but the rest of the paper is in another language).

- Scope: Here we analysed the content discussed in the papers. Papers were excluded due to scope because they did not specifically deal with adaptive gamification in learning. For example papers that discussed adaptive gamification for health or sport were removed.

- Duplicates: A few references were found in multiple databases, as some of the databases contain references to papers that are cited by papers that 
they publish. Furthermore some of the papers found were extended versions of previous papers. The non extended versions were therefore excluded.

After this filtering, we were left with a final total of twenty papers that we included in our final analysis.

\section{Literature analysis}

We analysed the papers through the lenses of each of our three research questions. We first identify the type of the contribution (section 3.1) to identify the degree of maturity of the research field. We then present the different adaptation systems by identifying what they are based on, and what they adapt (section 3.2). We more particularly distinguish static and dynamic adaptation as they rely on different mechanisms and different kinds of information. Finally we review the results of studies on the impact of the adaptation of game elements on learners' motivation and performances (section 3.3).

\subsection{Contributions: Recommendations, Architectures and Studies}

We examined the degree of maturity of the research field in light of two criteria. First, we identified the contribution type of each reviewed paper (table 2). Second, we reviewed the vocabulary used to describe the adapted content in each contribution.

Table 2. Type of each contribution: Recommendations, Architecture, or Study. These types are described below.

\begin{tabular}{|c|c|c|}
\hline \multirow{3}{*}{$\begin{array}{l}\text { Contribution } \\
\text { type }\end{array}$} & Recommendations $\delta$ & {$[2,4,6,9,10,11,21,23]$} \\
\hline & Architecture & $2[22,29]$ \\
\hline & Study & $10[18,19,20,25,28,30,31,34,35,36]$ \\
\hline
\end{tabular}

Regarding the first criterion, we classify the papers into three types of contributions that emerged from the review:

- Recommendations: identification of game elements that would be adapted to different categories or classes of learners, based on literature review, or general surveys ( 8 papers). These recommendations correspond to preliminary research and they have not been implemented in a system yet.

- Architectures: adaptation engines based on existing theoretical works, that have not yet been tested in real world situations (2 papers).

- Adaptation studies: an adaptation engine, based on recommendations to adapt game elements to learners, tested with learners through a real world study (the combination of an adaptation architecture, theoretical recommendations, and a real world study) (10 papers). 
Recommendations: We found two major categories of papers: papers that base their recommendations on literature surveys, and those that base their recommendations on user surveys, or feedback. In the first category, Borges et al. [4] review literature on "player types" (archetypal reasons why users seek out game experiences) and link these to learner roles and different game elements based on the motivational aspects they provide. Challco et al. [6] also link motivational aspects with player types and game elements. Škuta et al. [23] also use player types, but link them to higher level game principles. They then propose a matrix that associates game elements to player types based on how well each game element implements the linked game principles. In the second category, Denden et al. present three user studies, two based on a feedback after using a non adapted gamified tool $[9,11]$, and one based on a user survey [10] where participants rated statements based on game elements in order to determine their preference. Knutas et al. [21] analysed videos and interviews with learners in a software engineering project to create clusters of learners based on their interactions. These clusters were then linked to Bartle player types and relevant game elements. Barata et al. [2] used a similar approach, creating four types of learners based on their strategies during an online course. They then propose different goals that could be provided to each of the learner types. These studies serve to provide valuable information about what game elements learners might prefer, but still need to be implemented and tested in a real adaptation system.

Architectures: We found only two papers that describe adaptation engine architectures without any associated study. They present what the engine takes into account, what it adapts, and how it adapts it. Kuntas et al. [22] describe their process for designing an algorithm based personalised gamification system. They detail learner characteristics on which they base the adaptation of some game elements and the algorithm used to link the two. Monterrat et al. [29] describe an architecture that presents game elements as "epiphytes", completely separate from the learning content. They can therefore swap out game elements as needed. They also propose a module that tracks learner interactions in order to more finely adapt the game elements. They use a learner model that contains data on learner (gender, age, player type), usage data, and environment data.

Studies: Half of the reviewed papers present studies that rely on an adaptive gamification system in an educational setting $[18,19,20,25,28,30,31,34,35,36]$. These papers provide valuable results about the impact of adaptive gamification on learner motivation and performance. We present them in section 3.3.

Vocabulary: Regarding the second criterion, we observed that the papers reviewed have a general consensus about the vocabulary used to describe the gamification elements. Twelve of the papers reviewed [2,4,6,9,10,11,18,19,20,23,34,35] used the term "game element" to describe the low level implementations they 
use, such as points, levels, leaderboards, progress. Four papers from the same authors $[25,28,29,30]$ use the term "game features" to present the same level of implementation. Knutas et al. [21,22] use the terms "game like elements". Mora et al. [31] present different gamification "situations" (that combine different game elements). We can therefore observe that the papers reviewed generally agree on the term "game element" to designate what is adapted.

In summary, we find the field of adaptive gamification in education to be emergent, as there is a relatively low number of papers, that cover a wide variety of contribution types. Regarding the kind of contributions, twelve papers (two architectures and ten studies) take advantage of the ground work that the eight recommendations papers lay out. Furthermore, we found the vocabulary used to describe what is adapted to be quite stable, pointing towards a general consensus among authors.

\subsection{Information used for adaptation and its effect on game elements}

In this section we analyse both 1) what information is considered for adaptation (learner profile or activity) and 2) what the effect of the adaptation is (a change of the game element, or a modification of how the game element works). Our review analysis also allowed us to identify two major types of systems: static systems, and dynamic systems (see table 3). In a static system, the adaptation occurs once, usually before the learners start using the learning environment. In a dynamic system, the adaptation happens multiple times during the learning activity. We present our analysis according to these two categories as information considered for adaptation and its effect clearly depends on them.

Table 3. Classification of the papers according to the kind of information used for adaptation (user profile and/or activity), its effect (game element change or modification of its functioning) and the kind of adaptation (static or dynamic). The learner activity concerns either context based performance, or general behaviours. Some papers use multiple types of information, and are present on multiple rows.

\begin{tabular}{|c|c|c|c|c|c|}
\hline & \multicolumn{2}{|l|}{ Static } & \multirow{2}{*}{\multicolumn{2}{|c|}{$\begin{array}{c}\text { Dynamic } \\
\text { Change Modification }\end{array}$}} \\
\hline & & Change & Modification & & \\
\hline \multirow{4}{*}{ Profile } & Player Type & $8[4,6,23,25,28,30,31,36]$ & 0 & $2[21,29]$ & 0 \\
\hline & Personality & $4[9,11,18,35]$ & 0 & 0 & 0 \\
\hline & Expertise & $1[4]$ & 0 & 0 & 0 \\
\hline & Other & $2[4,10]$ & 0 & $1[29]$ & $1[22]$ \\
\hline \multirow{2}{*}{ Activity } & Performance & 0 & 0 & & $2[19,20]$ \\
\hline & Behaviours & 0 & 0 & $2[21,29]$ & $4[2,22,34]$ \\
\hline
\end{tabular}


Static adaptation Systems that use static adaptation all work in a similar manner. They base their adaptation on a learner profile, and adapt by changing game elements. Learners' profiles are identified, learners are sorted into different categories based on these profiles, and different game elements are given to each of the different categories of learners.

For learner profiles, the static adaptation systems generally use player types and more rarely learner personality. Player types are archetypal reasons or motivations that explain why players play games. The papers reviewed used either the Bartle Player types [3] (used in two papers [6,23]), the Brainhex player satisfaction model [32] (used in three papers [25, 28,36]), the Hexad player types [37] (used in one paper [31]), or the categories of players described by Ferro et al. [14] (used in one paper [4]). These different categorisations of players types describe the reasons why players prefer different games. For example the Hexad player classification describes "Achievers" as people who "like to prove themselves by tackling difficult challenges" [37]. The papers that use these player types typically use the definitions of the different categories as a basis for their adaptation rules, for example the Hexad classification suggests using badges and levels (amongst others) for Achievers. Brainhex and Hexad provide a questionnaire to determine a player profile, i.e a set of values that define how well the player fits each type. Generally studies adapt using the dominant player type, i.e. the type that scores the highest for a given learner. However, Mora at al. [31] question the precision of only using the dominant type and propose to consider several dimensions of the profile to tailor gamification.

For the personality traits, two of the five papers $[9,11]$ used the Big Five Factors personality traits [15]. Two papers used a user motivation questionnaire: Roosta et al. [35] used the framework presented by Elliot et al. [13]; Hassan et al. [18] used the questionnaire developed by Chen et al. [7]. Only a few static systems used other kinds of user characteristics, such as gender and gaming frequency [10], or learner role (tutor or tutee) [4].

Dynamic adaptation In dynamic adaptation, systems use learner activity to adapt game elements, either alone or in combination with a learner profile.

Systems that only use learner activity make adaptation by modifying the functioning of the game element. Two papers adapt the goals presented to learners. Paiva et al. [34] categorise all learner actions as either collaborative, gamification, individual or social interactions; the system adapts the kind of goals the learner receives according to the kind of actions they perform. Barata et al. [2] propose a system that varies the goals and rewards given to learners based on their behaviours, by distinguishing four types of learners: achievers, disheartened, underachievers, and late bloomers (a learner is not fixed into a specific category, as their behaviour may vary over time). Jagušt et al. [19] present two dynamic adaptation situations, both of them using learner activity. In the first situation, learners are timed in a maths quiz. Each time the learner gets a question right, they are given less time for the next question, essentially increasing the difficulty based on the learner's performance. In the second situation, the 
learners are shown a target score that changes depending on how they respond to questions: the more correct answers they give, the more the target score increases. Kickmeier-rust et al. [20] change the types of badges presented to, and feedback received by the learner based on the mistakes they make.

Two systems use both learner activity and profile. Monterrat et al. [29, 30] aim to modify the learners' profile based on their activity. The system then uses previously established static adaptation rules. When the learners' profile changes significantly, a different game element is given to the learner. The learner profile is based on the Ferro player types in earlier versions of their work [29], and in more recent work [30] they propose to use the Brainhex model (in [29] they also use gender and age for adaptation). This is a straight forward way of implementing dynamic adaptive gamification using static adaptation rules. The systems proposed by Knutas et al. [21,22] use an algorithm that also uses learners' profile and interactions. In both systems, they use the Hexad player profile, and in the more recent one [22] they also use learner skills. In [21] they analysed videos of students during project meetings and classified their interactions and propose different game elements based on a combination of profile and interaction types. They lay the ground rules for a dynamic adaptation based on learner activity, but do not offer a method to detect these actions in real time. In [22] they use learner chat activity and profile to provide personalised goals.

In summary, adaptation of game elements is made using two major categories of information: static adaptation mainly relies on learners' profile (mainly their preferences and motivations), dynamic adaptation is based on how learners perform with regards to the learning content, or how the learners interact with the system in general. The majority of systems then use this information to select which game elements would be the most appropriate for learners. Only a few (five) adapt by modifying how the game elements function.

\subsection{Impact of adapted gamification on learners}

We examined the impact of adaptive systems reported in the "study" papers identified in section 3.1. We found that the results could be split into two categories (see Table 4) those that show a general positive impact on learner's motivation or performance, and 2) those that show more mitigated results. We also split the studies based on 1) whether they used a static or dynamic adaptation, and 2) the duration to investigate whether these factors influence the impact of adaptive gamification on learners. We identified short studies as those lasting less than two weeks, and long studies as lasting more than two weeks (with an experimental process that is closer to real world learning practices).

Short studies We found two studies that lasted less than two weeks [19,20], with both of these studies using a dynamic adaptation. All of these studies reported positive results on learners. In [20], learners used the adaptive system over two sessions, for a total possible time of thirty minutes. According to the 
Table 4. Impact of the reviewed studies. The numbers show how many studies are present in each category.

\begin{tabular}{|c|c|c|c|}
\hline \multirow[t]{2}{*}{ Duration } & \multicolumn{2}{|c|}{ Static } & \multirow{2}{*}{$\mid \begin{array}{c}\text { Dynamic } \\
\text { Positive Mitigated }\end{array}$} \\
\hline & Positive & Mitigated & \\
\hline Short & 0 & 0 & $2[19,20] 0$ \\
\hline Long & $4[18,28,31,35]$ & $2[25,30]$ & $1[34]$ \\
\hline
\end{tabular}

authors the personalised system reduced the amount of errors that learners made. Learners with the adaptive situation showed a larger decrease in errors made in the second session when compared to learners that used the non adaptive situation. In [19] Jagust et al. test two adaptive situations that learners used for 15 minutes each. In the first situation, the time learners had to answer questions changed depending on how quickly they answered the previous question. In the second situation, a target score changed depending on group performance. In both situations the authors report an increase in learner performance (learners completed more tasks than compared to a non gamified situation), although the first situation caused a larger increase than the second one.

Long studies Seven of the reviewed studies lasted more than three weeks [18, $25,28,30,31,34,35]$. Four studies showed generally positive results $[18,28,31,35]$. Roosta et al. [35] presented learners with a different game element based on their motivation type. Learners used an online tool for one month. The authors find that learners who had game elements that were suited to their motivation type showed significant differences in motivation, engagement, and quiz results when compared to learners who had randomly assigned game elements. They used learner participation rates in the online activities as a metric to gauge motivation and engagement. Monterrat et al. [28] split learners into three different groups: one group received game elements adapted to their Brainhex player type, one group received counter-adapted game elements, and the third group received random game elements. Learners were then free to use the learning environment as they wanted over a three week period. The authors found that learners with the adapted game elements spent more time using the learning tool that those with the counter adapted elements. Hassan et al. [18] also showed a widely positive result in their study: learners who used game elements adapted to their learning style showed a higher course completion rate than those who used random game elements. This impact was also observed with learners' self-reported motivation using a questionnaire. Finally Mora et al. [31] also report a general positive impact from their adaptation, with an increase in behavioural and emotional engagement in learners, reported using a questionnaire that was given to learners after using the tool. In this study, university learners were sorted into different groups based on their Hexad profile (the groups contained users that had similar Hexad profiles) and used a learning tool over a period of 14 weeks, with each of the different Hexad groups receiving different game elements. How- 
ever, the authors themselves point out that these results are not significant due to the small sample size.

The other three studies showed more mitigated results [25,30,34]. In Monterrat et al. [30] learners used the learning environment during 3 structured learning sessions, each lasting 45 minutes set over a three week period. The learners were middle school students, and used the learning environment as normal part of their lessons. The results show that learners with counter-adapted game elements found their game elements to be more fun and useful than learners with adapted or random elements. The authors performed a similar study reported in [25], with adults who used the learning tool voluntarily. Learners were free to use the learning tool over three weeks. They found little to no difference for the majority of learners. They found that adaptation had an influence only on the more invested learners: learners with adapted game elements showed less amotivation (calculated using a questionnaire [16]). They did not find any difference in learner enjoyment for those particular learners. Paiva et al. [34] analysed the usage data during the month after the introduction of tailored goals in their learning tool. Learners received personalised goals to encourage them to increase the number of specific learning actions they performed (for example learners who performed a low number of individual learning actions were shown goals designed to increase their number of individual learning actions). The authors found that the social and collaborative goals were effective in increasing the number of related actions. However this effect was not observed with individual learning goals (they do not observe an increase in the number of individual learning actions).

In summary we can see that shorter studies tend to show positive results from adaptive gamification, where as the longer ones show more mitigated results. The two short studies compared the impact of the adaptive gamified situation to a non adaptive gamified situation, this does not allow us to understand if the impact on learners is due to the adaptive nature of the gamified system, or due to the introduction of a novel gamified system itself. With the longer studies, we can assume that the novelty effect wears off, thus leading to more mitigated results, as the static adaptation tested in the longer studies may not be precise enough to take learner variations into. This novelty effect was also identified by Hamari et al. in [17]. Furthermore, we can see that there is some contradictory results from the different papers. [28] and [18] both report an increase in learner motivation for all learners in their studies, whereas [25] only show an increase in the more invested learners. This could be due to the nature of the metrics used to gauge learner motivation. In [18] they use a questionnaire to establish this, but $[25,28]$ both use the time learners spent using the tool.

\section{Future research agenda}

Adaptive gamification in education is a novel and cutting edge research field, that has been gaining in popularity in the past few years. In order to better understand the current state of research in this field we performed an in-depth 
literature review that included twenty papers. Our analysis highlights a strong theoretical base, with eight papers that present recommendations for game elements, two that propose architectures that use these recommendations, and ten papers that test various adaptation engines in real world learning settings. We observed a variety of information used as a basis for adaptation, with both static and dynamic approaches to adaptation. This shows that this is a wide and diverse research field. In order to guide future research, we present three needs that emerge from our literature analysis that should be addressed in the future.

\subsection{The need for richer learner models}

As pointed out in 3.2, half of the reviewed papers use learner player types to adapt game elements. Generally they use the dominant player type identified to classify the learners. Mora et al. [31] question this in their study and show promising results when adapting to more than the dominant player type (although as the authors state, their results are not significant). Furthermore very few systems (only two) take learning characteristics into account, such as learner expertise [4] or learning styles [18]. We believe that the mitigated results identified in 3.3 could be partly due to the complex nature of learner preferences that are not represented in these simplified learner classifications. We therefore firstly advise taking into account more complex learner profiles, that include more specific learning data, such as learner expertise, learner skills as well as learner player types. Furthermore, learner activity should also be better explored as a means for adapting game elements.

\subsection{The need to explore different adaptation methods}

We identified in section 3.2 how adaptation of gamification may affect the gamified learning environment by changing the game element itself, or by modifying its functioning. In their current state, most adaptation systems work in a static way. We highly believe that there is more to be explored in the domain of dynamic adaptation. For example the question of how and when a dynamic adaptation presents itself to a learner still has to be addressed. If the change brought on by the adaptation is not explained or presented to the learner in a clear and understandable manner this could confuse and could distract the learner from his/her learning activity. In the field of user interface adaptation Bouzit et al. [5] show that change needs to be observable, intelligible, predictable and controllable for the user. We believe therefore that research needs to be done into how these concepts can be applied to educational settings.

\subsection{The need for longer and more structured studies}

As identified in section 3.3, we advise that future adaptive gamification studies should aim for longer durations, as the results from short studies may be affected by the novelty effect of introducing gamification and not the adaptive nature of 
the gamified system. Furthermore, studies should compare the effectiveness of the adaptive system to that of a non adaptive system, which would also help with identifying if the impact on learners is due to gamification in general or to the adaptive nature. We also observed two ways for studies to quantify the effectiveness of the tested systems: either as an impact on learner performance or learner motivation. For learner performance it is fairly straightforward, using metrics such as course completion rate [18], or test results [20]. However, for learner motivation, the process was some-what more complex, as studies used ad-hoc metrics to infer learner motivation (for example [25] used time spent on the learning tool, [30,34] used learner feedback). This makes the comparison of the results from different studies difficult to make. We therefore advise that more research be performed into a more structured manner to estimate learner motivation levels.

\section{Conclusion}

In this paper we presented an in depth literature review in order to better understand the field of adaptive gamification in education. We identified that the field is emergent, with a theoretical base that several studies in real world learning settings build upon, and a general consensus on the language used. There is still room for this field to grow and develop, especially regarding dynamic adaptation that has been studied only once on a long term. We listed three needs that should be fulfilled in future research, based on the shortcomings we have identified. First, we highlighted the need for richer learner models that adaptation systems can use for adaptation. Second, dynamic adaptation methods should be deepened to better adapt to learner behaviour. Third, there is a need for longer and more structured studies in order to better understand and be able to compare the impact of adaptive systems on learners.

\section{Acknowledgements}

This work is a part of the LudiMoodle project financed by the e-FRAN Programme d'investissement d'avenir, operated by the Caisse des Dépots.

\section{References}

1. Attali, Y., Arieli-Attali, M.: Gamification in assessment: Do points affect test performance? Computers \& Education 83 (Apr 2015)

2. Barata, G., Gama, S., Jorge, J., Gonçalves, D.: Gamification for smarter learning: tales from the trenches. Smart Learning Environments p. 10 (2015)

3. Bartle, R.: Hearts, clubs, diamonds, spades: Players who suit MUDs. Journal of MUD research (1), 19 (1996)

4. Borges, S.S., Mizoguchi, R., Durelli, V.H.S., Bittencourt, I.I., Isotani, S.: A link between worlds: Towards a conceptual framework for bridging player and learner roles in gamified collaborative learning contexts. In: Advances in Social Computing and Digital Education. pp. 19-34. Springer (2016) 
5. Bouzit, S., Calvary, G., Coutaz, J., Chêne, D., Petit, E., Vanderdonckt, J.: The PDA-LPA design space for user interface adaptation. In: 2017 11th International Conference on Research Challenges in Information Science (RCIS). pp. 353-364 (2017)

6. Challco, G.C., Moreira, D.A., Mizoguchi, R., Isotani, S.: An ontology engineering approach to gamify collaborative learning scenarios. In: Collaboration and Technology. pp. 185-198. Lecture Notes in Computer Science, Springer (2014)

7. Chen, K.C., Jang, S.J.: Motivation in online learning: Testing a model of selfdetermination theory. Computers in Human Behavior 26(4), 741-752 (2010)

8. De Bra, P., Aerts, A., Berden, B., De Lange, B., Rousseau, B., Santic, T., Smits, D., Stash, N.: AHA! The adaptive hypermedia architecture. In: Proceedings of the fourteenth ACM conference on Hypertext and hypermedia. pp. 81-84. ACM (2003)

9. Denden, M., Tlili, A., Essalmi, F., Jemni, M.: Educational gamification based on personality. In: 2017 IEEE/ACS 14th International Conference on Computer Systems and Applications (AICCSA). pp. 1399-1405 (2017)

10. Denden, M., Tlili, A., Essalmi, F., Jemni, M.: An investigation of the factors affecting the perception of gamification and game elements. In: 2017 6th International Conference on Information and Communication Technology and Accessibility (ICTA). pp. 1-6 (2017)

11. Denden, M., Tlili, A., Essalmi, F., Jemni, M.: Does personality affect students' perceived preferences for game elements in gamified learning environments? In: 18th International Conference on Advanced Learning Technologies (ICALT). pp. 111-115. IEEE (2018)

12. Deterding, S., Dixon, D., Khaled, R., Nacke, L.: From game design elements to gamefulness: defining gamification. In: Proceedings of the 15th international academic MindTrek conference: Envisioning future media environments. pp. 9-15. ACM (2011)

13. Elliot, A.J., Murayama, K.: On the measurement of achievement goals: Critique, illustration, and application. Journal of educational psychology 100(3), 613 (2008)

14. Ferro, L.S., Walz, S.P., Greuter, S.: Towards personalised, gamified systems: An investigation into game design, personality and player typologies. In: Proceedings of The 9th Australasian Conference on Interactive Entertainment: Matters of Life and Death. pp. 7:1-7:6. ACM (2013)

15. Goldberg, L.R.: An alternative "description of personality": the big-five factor structure. Journal of personality and social psychology 59(6), 1216 (1990)

16. Guay, F., Vallerand, R.J., Blanchard, C.: On the assessment of situational intrinsic and extrinsic motivation: The situational motivation scale (sims). Motivation and emotion 24(3), 175-213 (2000)

17. Hamari, J., Koivisto, J., Sarsa, H.: Does gamification work? - a literature review of empirical studies on gamification. In: 47th Hawaii International Conference on System Sciences. pp. 3025-3034 (2014)

18. Hassan, M.A., Habiba, U., Majeed, F., Shoaib, M.: Adaptive gamification in elearning based on students' learning styles. Interactive Learning Environments pp. 1-21 (2019)

19. Jagušt, T., Botički, I., So, H.J.: Examining competitive, collaborative and adaptive gamification in young learners' math learning. Computers \& Education 125, 444457 (2018)

20. Kickmeier-Rust, M.D., Hillemann, E.C., Albert, D.: Gamification and smart feedback: Experiences with a primary school level math app. Int. J. Game-Based Learn. 4(3), 35-46 (2014) 
21. Knutas, A., Ikonen, J., Maggiorini, D., Ripamonti, L., Porras, J.: Creating student interaction profiles for adaptive collaboration gamification design. Int. J. Hum. Cap. Inf. Technol. Prof. 7(3), 47-62

22. Knutas, A., van Roy, R., Hynninen, T., Granato, M., Kasurinen, J., Ikonen, J.: A process for designing algorithm-based personalized gamification. Multimedia Tools and Applications (2018)

23. Škuta, P., Kostolányová, K.: Adaptive Aproach to the Gamification in Education. DIVAI 2018 (2018)

24. Landers, R.N., Armstrong, M.B.: Enhancing instructional outcomes with gamification: An empirical test of the Technology-Enhanced Training Effectiveness Model. Computers in Human Behavior (Sep 2015)

25. Lavoué, E., Monterrat, B., Desmarais, M., George, S.: Adaptive gamification for learning environments. IEEE Transactions on Learning Technologies 12(1), 16-28 (2018)

26. Lopez, C., Tucker, C.: Towards personalized adaptive gamification: A machine learning model for predicting performance. IEEE Transactions on Games (2018)

27. de Marcos, L., Garcia-Lopez, E., Garcia-Cabot, A.: On the effectiveness of gamelike and social approaches in learning: Comparing educational gaming, gamification \& social networking. Computers \& Education 95 (2016)

28. Monterrat, B., Desmarais, M., Lavoué, E., George, S.: A player model for adaptive gamification in learning environments. In: Artificial Intelligence in Education. pp. 297-306. Springer (2015)

29. Monterrat, B., Lavoué, E., George, S.: Toward an adaptive gamification system for learning environments. In: Computer Supported Education. pp. 115-129. Springer (2015)

30. Monterrat, B., Lavoué, E., George, S.: Adaptation of gaming features for motivating learners. Simulation \& Gaming 48(5), 625-656 (2017)

31. Mora, A., Tondello, G.F., Nacke, L.E., Arnedo-Moreno, J.: Effect of personalized gameful design on student engagement. In: IEEE Global Engineering Education Conference (EDUCON). pp. 1925-1933 (2018)

32. Nacke, L.E., Bateman, C., Mandryk, R.L.: BrainHex: A neurobiological gamer typology survey. Entertainment Computing 5(1), 55-62 (2014)

33. Orji, R., Nacke, L.E., DiMarco, C.: Towards personality-driven persuasive health games and gamified systems. In: Proceedings of SIGCHI Conference on Human Factors in Computing Systems (2017)

34. Paiva, R., Bittencourt, I.I., Tenório, T., Jaques, P., Isotani, S.: What do students do on-line? modeling students' interactions to improve their learning experience. Computers in Human Behavior 64, 769-781 (2016)

35. Roosta, F., Taghiyareh, F., Mosharraf, M.: Personalization of gamificationelements in an e-learning environment based on learners' motivation. In: 8th International Symposium on Telecommunications (IST). pp. 637-642 (2016)

36. dos Santos, W.O., Bittencourt, I.I., Vassileva, J.: Gamification Design to Tailor Gamified Educational Systems Based on Gamer Types. In: SBGames 2018 (2018)

37. Tondello, G.F., Wehbe, R.R., Diamond, L., Busch, M., Marczewski, A., Nacke, L.E.: The gamification user types hexad scale. In: Symposium on Computer-Human Interaction in Play. pp. 229-243. ACM (2016)

38. Vom Brocke, J., Simons, A., Niehaves, B., Riemer, K., Plattfaut, R., Cleven, A.: Reconstructing the giant: on the importance of rigour in documenting the literature search process. In: ECIS Proceedings 161. vol. 9, pp. 2206-2217 (2009)

39. Webster, J., Watson, R.T.: Analyzing the past to prepare for the future: Writing a literature review. MIS quarterly pp. xiii-xxiii (2002) 